\title{
Determination of errors that compromise the quality of laboratory service in a tertiary hospital
}

\author{
Kenneth Kipruto Kimengech'1, Stanley Kinge Waithaka², Jackson Onyuka², \\ Christian Sekadde Kigondu ${ }^{3}$ \\ ${ }^{1}$ Assistant Chief Medical Laboratory Officer, Department of Laboratory Medicine, Clinical Chemistry Laboratory \\ Kenyatta National Hospital, Nairobi-Kenya, ${ }^{2}$ Lecturer, Health Sciences Department of Medical Sciences Mount Kenya \\ University, ${ }^{3}$ Professor, Clinical Chemistry Thematic Area of Clinical Chemistry, Department of Human Pathology, School \\ of Medicine, College of Health Sciences University of Nairobi, Kenya
}

Background: Clinical Laboratory testing is a highly complex process that entails numerous procedures. Although it has been known that laboratory testing services are safe, it is increasingly becoming a common knowledge that they are not that safe. Studies have indicated that there are a number of errors that occur due to laboratory testing processes. These errors may not be realized easily during the testing process, but they make significant impact on the results given. Aims and Objective: To determine the levels of pre-analytical, analytical, and post analytical errors found in the analysis of Clinical chemistry Laboratory specimen. Materials and Methods: A prospective and Descriptive study was carried out at Clinical Chemistry a total of 346 request forms, specimens/samples and dispatched results were scrutinized and errors documented as per the different variables in the different phases, over a period of three months and the findings were analyzed. Results: Results of the study showed that Preanalytical errors were most common with a frequency of $148(42.8 \%)$, followed by analytical errors 114 (32.9\%) and post analytical errors 84 (24.3\%), respectively. Conclusions: The study concludes that pre-analytical, analytical, and post analytical errors are errors that compromise the quality of laboratory service delivery, which impacts on the patient management and diagnosis. Clinical laboratory errors can be minimized if due diligence and professionalism is adhered in the laboratory.

Key words: Clinical, Laboratory, Errors, Quality, Processes

\section{INTRODUCTION}

Clinical Laboratory testing is a highly complex process that entails numerous procedures. Although it has been known that laboratory testing services are safe, it is increasingly becoming a common knowledge that they are not that safe. Studies have indicated that there are a number of errors that occur due to laboratory testing processes. These errors may not be realized easily during the testing process, but they make significant impact on the results given. Consequently, patients may not be treated according to the conditions or diseases they are suffering from. Thus, it complicates the process of disease management. ${ }^{1}$

Non-conformance analysis involves the recognition of errors or deviations from standard or accredited practice. ${ }^{2}$ Identification of non-conformance's in a medical laboratory is an essential component of accreditation to the International Standards from the international Organization for Standardization. ${ }^{3}$

Over the past five years, efforts have been made to improve laboratory services in Africa. In January 2008, the consensus 
meeting on Clinical Laboratory Testing Harmonization and standardization convened governments, agencies and development partners in Maputo, Mozambique. Subsequent meetings held in 2008 on African Laboratory Medicine in Lyon, France, Yaoundé, Cameroon and Dakar, Senegal formulated strategies for Laboratory Strengthening and arrived at several landmark achievements. ${ }^{4}$

In 2009, the World Health Organization, Regional Office for Africa (WHO-AFRO) and partners launched the Stepwise Laboratory Quality Improvement Process Towards Accreditation. ${ }^{5}$ To help laboratories in resourcelimited settings strive toward international accreditation. ${ }^{6}$

The major mistakes in laboratory diagnostics arise during patient preparation, sample collection, sample preparation and sample storage. Most of these errors are due to the initial procedures of the testing process carried out by the healthcare personnel outside the laboratory walls and outside the direct control of the Clinical laboratory. A laboratory error is defined as a defect occurring at any part of the laboratory cycle, from ordering tests to reporting results and appropriately interpreting and reacting in these. According to these concepts, some practical considerations should be made in order to reduce errors in laboratory medicine and improve patient safety. ${ }^{1}$

\section{MATERIAL AND METHODS}

A total of 346 request forms, specimens/samples and dispatch of results were scrutinized and errors documented as per the different variables in the different phases, over a period of three months prospective study at the Clinical Chemistry Laboratory.

\section{RESULTS}

The study findings indicate that the pre-analytical phase of the Clinical laboratory testing process had 148 errors which were $42.8 \%$ of the total number of errors captured during the study period. $74.3 \%$ (110) of the pre-analytical errors were attributed to: request forms lacking address $(40,27.0 \%)$; test not done in biochemistry lab $(24,16.2 \%)$; specimen drawn in wrong tube $(20,13.5 \%)$; specimen without request forms $(10,6.8 \%)$; unlabeled specimen $(8,5.4 \%)$; and inadequate/insufficient sample after centrifugation $(8,5.4 \%)$. The remaining $25.7 \%$ (38) of the pre-analytical phase errors are associated to: no patient name or identification number $(7,4.7 \%)$; contaminated specimen bottle $(6,4.1 \%)$; mismatched information on request form and specimen container $(5,3.4 \%)$; no specimen received yet there is request form $(5,3.4 \%)$; specimen not paid for $(5,3.4 \%)$; leaking specimen/broken container $(3,2.0 \%)$; wrong specimen $(3,2.0 \%)$; specimen clotted yet test requires unclotted specimen $(2,1.4 \%)$; test not well specified $(1,0.7 \%)$; and wrong test compared to the container type $(1,0.7 \%)$.

\subsection{Pre-Analytical Phase Errors}

The study findings indicate that the pre-analytical phase of the Clinical laboratory testing process had 148 errors (Figure 1) which were $42.8 \%$ of the total number of errors captured during the study period. $74.3 \%$ (110) of the preanalytical errors were attributed to: request forms lacking address (40,27.0\%); test not done in biochemistry lab $(24,16.2 \%)$; specimen drawn in wrong tube $(20,13.5 \%)$; specimen without request forms $(10,6.8 \%)$; unlabeled specimen $(8,5.4 \%)$; and inadequate/insufficient sample after centrifugation $(8,5.4 \%)$. The remaining $25.7 \%$ (38) of the pre-analytical phase errors are associated to: no patient name or identification number $(7,4.7 \%)$; contaminated specimen bottle (6, 4.1\%); mismatched information on request form and specimen container $(5,3.4 \%)$ this could be attributed as a result of manual registration as shown in Plate 1; no specimen received yet there is request form (5, $3.4 \%)$; specimen not paid for $(5,3.4 \%)$; leaking specimen/ broken container $(3,2.0 \%)$; wrong specimen $(3,2.0 \%)$; specimen clotted yet test requires unclotted specimen $(2$, $1.4 \%)$; test not well specified $(1,0.7 \%)$; and wrong test compared to the container type $(1,0.7 \%)$. The pre-analytical phase results are summarized in Table 1.

Table 1; showed the overall percent of errors in the preanalytical phase. The total error frequency of 148 was a statistically significant $(P<0.05)$ in the error frequencies

\begin{tabular}{lcc}
\hline \multicolumn{3}{l}{ Table 1: Pre-Analytical Phase Errors } \\
\hline Attribute & Frequency & Percent \\
\hline Request forms lacking address & 40 & $27.0 \%$ \\
Test not done in Biochemistry Lab & 24 & $16.2 \%$ \\
Specimen drawn in wrong tube & 20 & $13.5 \%$ \\
Specimen without request forms & 10 & $6.8 \%$ \\
Unlabeled specimen & 8 & $5.4 \%$ \\
Inadequate/insufficient sample after & 8 & $5.4 \%$ \\
centrifugation & & \\
No patient name or identification & 7 & $4.7 \%$ \\
number & & \\
Contaminated specimen bottle & 6 & $4.1 \%$ \\
Mismatched information on request & 5 & $3.4 \%$ \\
form and specimen container & & \\
No specimen received yet there is & 5 & $3.4 \%$ \\
request form & & \\
Specimen not paid for & 5 & $3.4 \%$ \\
Leaking specimen/broken container & 3 & $2.0 \%$ \\
Wrong specimen received & 3 & $2.0 \%$ \\
Specimen clotted yet test requires & 2 & $1.4 \%$ \\
Whole blood specimen & & \\
$\quad$ Test not well specified (too broad) & 1 & $0.7 \%$ \\
Wrong test compared to the & 1 & $0.7 \%$ \\
container type & & \\
Total & 148 & $100.0 \%$ \\
\hline
\end{tabular}


observed in the pre-analytical phase which had the highest number of errors with 148 (42.8\% of total error).

The above photo shows manual registration of specimens being received in clinical chemistry automation reduces the variability of results and errors of analysis by eliminating tasks that are repetitive and monotonous for a human and that can lead to boredom or inattention.

Plate 2 shows samples already centrifuged with inconsistent volume of the samples, which later lead to inadequate or insufficient volume for analysis.

\subsection{Analytical Phase Errors}

The study findings indicate that the analytical phase of the Clinical laboratory testing process had 114 errors (Figure 1) noted, which is, $32.9 \%$ of the total number of errors captured during the study period, $81.6 \%$ (93) of the analytical errors were attributed to: reagent out of stock $(33,28.9 \%)$; inadequate sample during test runs (29, 25.4\%); haemolysed samples giving poor results (13, $11.4 \%$ ); duplication of lab number in different specimens $(12,10.5 \%)$; and IQC not done before test runs $(6,5.3 \%)$. The remaining $18.4 \%$ (21) of the analytical phase errors are associated to: icterus causing test interference as a factor (5, 4.4\%); analyzer failure during analytical (4, 3.5\%); contradicting results upon re-testing $(3,2.6 \%)$; improper sample storage conditions $(3,2.6 \%)$; wrong specimen analyzed $(2,1.8 \%)$; Lipemic causing test interference as a factor $(2,1.8 \%)$; name on specimen container and request form not tallying $(1,0.9 \%)$; and test requested not clear $(1,0.9 \%)$. The following errors were not reported at all in the analytical phase: calibration error; lab incident/ occurrence/ accidental spillage; EQA/PT not done; and IQC not done. The analytical phase results are summarized in Table 2.

In Table2; the overall percent of errors in the analytical phase. With a total error frequency of 114. There was a statistically significant difference (Chi-square, 155.5; df, $1 ; P<0.05)$ in the error frequencies observed in the analytical phase which had a significant high number of errors with 114 ( $32.9 \%$ of total error).

In Plate 3, samples already centrifuged and dispensed to sample cups shows visual evidence of hemolysis. Even slight hemolysis has effect on most of the Clinical Chemistry test values. For example concentrations of Aspartate aminotransferase, aldolase, lactate dehydrogenase, total acid phosphatase, isocitrate dehydrogenase, potassium, magnesium, and phosphate are particularly increased by hemolysis. Additional band caused by hemolysis may be observed on serum protein electrophoresis.

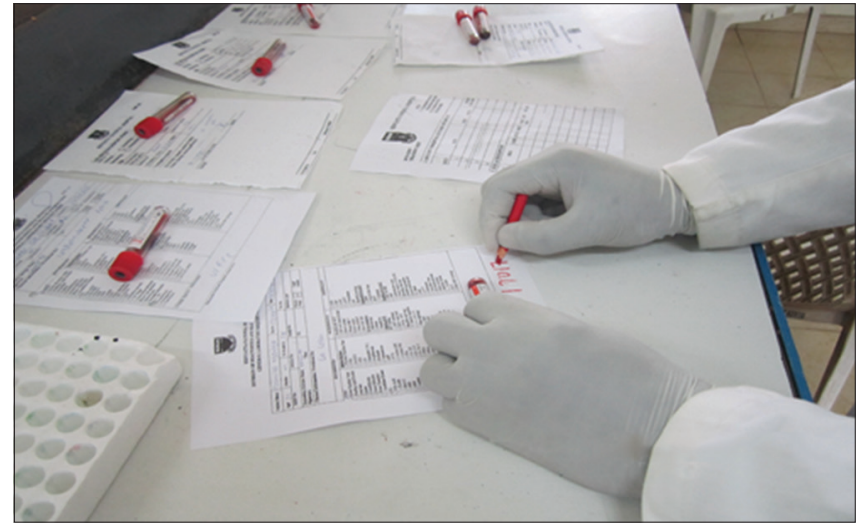

Plate 1: Manual registration of specimens

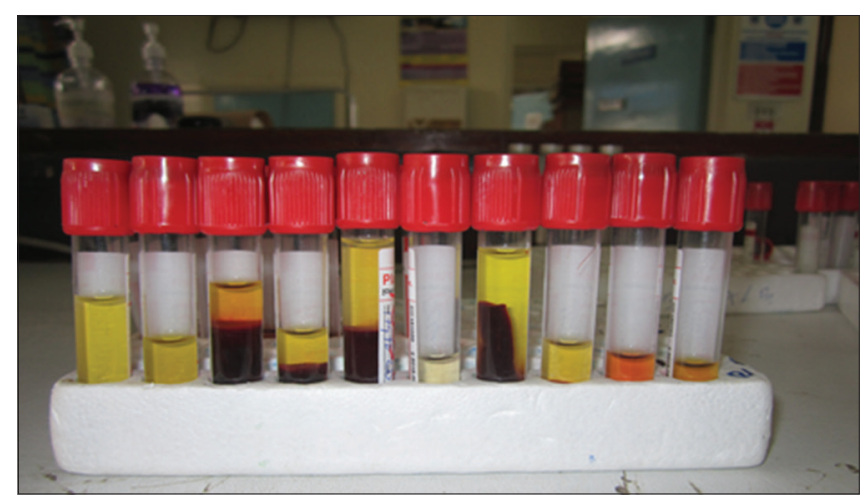

Plate 2: Specimens centrifuged

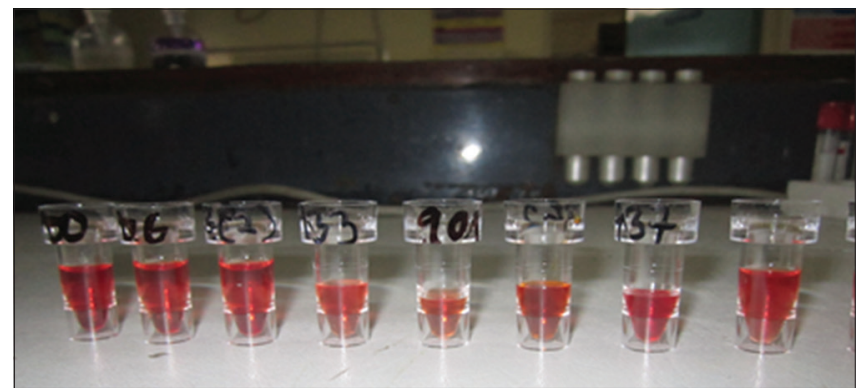

Plate 3: Samples grossly haemolysed

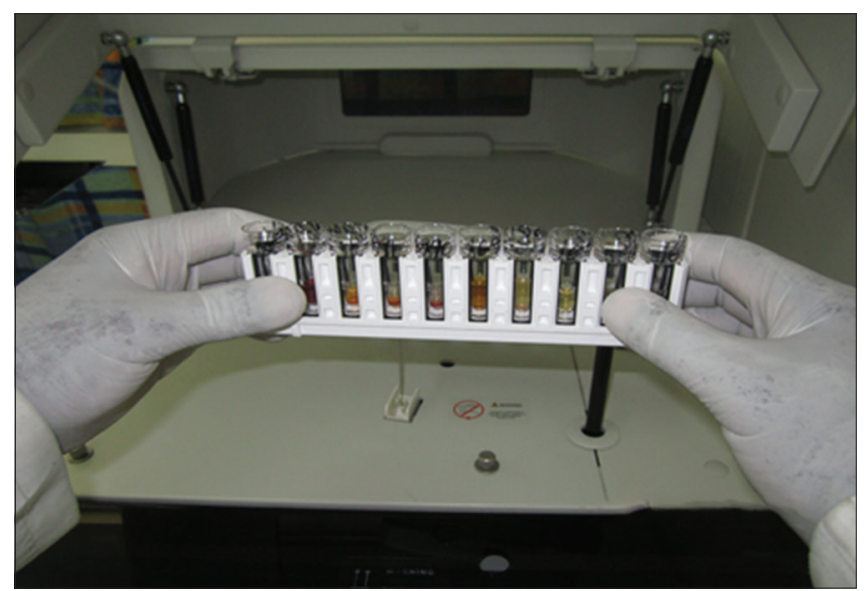

Plate 4: Insufficient serum loaded into the analyzer

Asian Journal of Medical Sciences | Jan-Feb 2017 | Vol 8 | Issue 
In Plate 4, insufficient samples being loaded to the Clinical Chemistry analyzer during the analytical phase. The insufficient samples constituted $25.4 \%$ of the analytical errors.

\subsection{Post-Analytical Phase Errors}

The study findings indicate that the post-analytical phase of the Clinical laboratory testing process had 84 (Figure 1) $(24.3 \%)$ errors noted, $82.1 \%$ (69) of the analytical errors were attributed to: uncollected results $(25,29.8 \%)$; critical values $(17,20.2 \%)$; results lost $(16,19.0 \%)$; and un-reviewed results $(11,13.1 \%)$. The remaining $18.4 \%$ (21) of the postanalytical phase errors are associated to: wrong test done on specimen (3, 3.6\%); none adherence to turn around time $(2,2.4 \%)$; not all requested tests done $(2,2.4 \%)$; results attached to wrong request form $(2,2.4 \%)$; illegible results $(1,1.2 \%)$; self request results not easy post $(1,1.2 \%)$; dispatched result printout without the actual results (1, $1.2 \%)$; results released without being reviewed $(1,1.2 \%)$; results released without request form which was found later $(1,1.2 \%)$; and specimen type not indicated on the request form $(1,1.2 \%)$. The following errors were not reported at all in the post-analytical phase: transcription error; results not posted; and leaking specimen/broken container. The post-analytical phase results are summarized in Table 3.

Table 3; showed the overall percent of errors in the postanalytical phase. With a total error frequency of 84 . There was a statistically significant difference (Chi-square, 198.4; $\mathrm{df}, 1 ; P<0.05)$ in the error frequencies observed in the post-analytical phase which had the lowest number of errors with 84 (24.3\% of total error).

All the pre-analytical, analytical and post analytical errors had an impact to the client or the patient either directly or indirectly, in terms of service delivery, quality health care and cost effect. Most of the patients $40(27.0 \%)$ did not get back their results due request forms lacking address i.e.

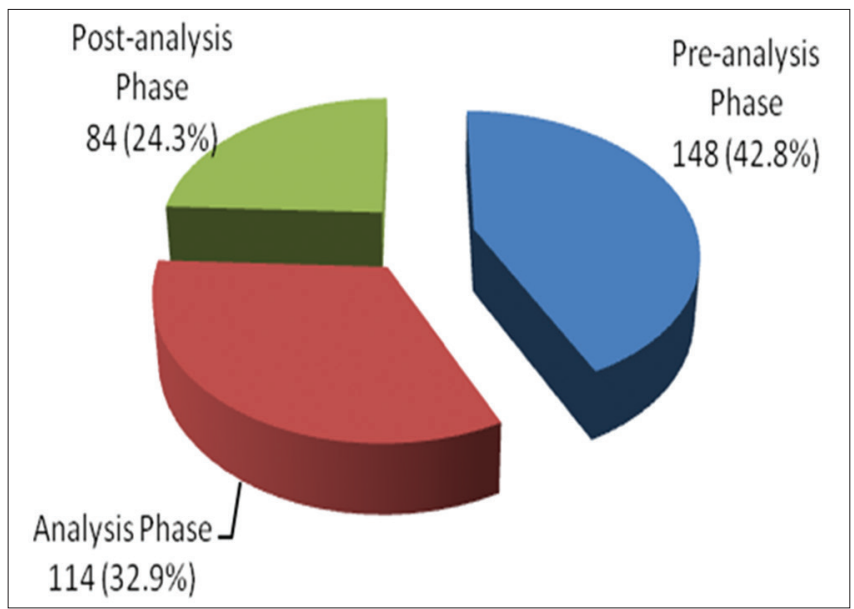

Figure 1: Errors distribution in relation to pre-analytical, analytical and post-analytical ward or clinic, and this prolonged the turnaround time for the results due to wasted time in tracing the address, some results took more than a month to reach the clinician or the patients file. And therefore this prolonged the patient stay in the Hospital and quality health care Management.

In the three months study a total of $8(5.4 \%)$ unlabeled specimen was received and rejected in the laboratory register. That means another specimen for the eight patients had to be recollected and brought again to the laboratory for analysis.

\section{DISCUSSION}

Most of the pre-analytical errors (110, 74.3\%) were attributed to request forms lacking address. Computerized

\begin{tabular}{|c|c|c|}
\hline Attribute & Frequency & Percent \\
\hline Reagent out of stock & 33 & $28.9 \%$ \\
\hline Inadequate sample during test runs & 29 & $25.4 \%$ \\
\hline $\begin{array}{l}\text { Haemolysed samples giving poor } \\
\text { results }\end{array}$ & 13 & $11.4 \%$ \\
\hline $\begin{array}{l}\text { Duplication of lab number in } \\
\text { different specimens }\end{array}$ & 12 & $10.5 \%$ \\
\hline IQC not done before test runs & 6 & $5.3 \%$ \\
\hline $\begin{array}{l}\text { Icterus causing test interference as } \\
\text { a factor }\end{array}$ & 5 & $4.4 \%$ \\
\hline Analyzer failure during analytical & 4 & $3.5 \%$ \\
\hline Contradicting results upon re-testing & 3 & $2.6 \%$ \\
\hline Improper sample storage conditions & 3 & $2.6 \%$ \\
\hline Wrong specimen analyzed & 2 & $1.8 \%$ \\
\hline $\begin{array}{l}\text { Lipemic causing test interference as } \\
\text { a factor }\end{array}$ & 2 & $1.8 \%$ \\
\hline $\begin{array}{l}\text { Name on specimen container and } \\
\text { request form not tallying }\end{array}$ & 1 & $0.9 \%$ \\
\hline Test requested not clear & 1 & $0.9 \%$ \\
\hline Total & 114 & $100.0 \%$ \\
\hline
\end{tabular}

\begin{tabular}{lcc} 
Table 3: Post-Analytical Phase Errors & \\
\hline Attribute & Frequency & Percent \\
\hline Uncollected results & 25 & $29.8 \%$ \\
Critical values & 17 & $20.2 \%$ \\
Results lost & 16 & $19.0 \%$ \\
Un-reviewed results & 11 & $13.1 \%$ \\
Wrong test done on specimen & 3 & $3.6 \%$ \\
None adherence to turn around time & 2 & $2.4 \%$ \\
Not all requested tests done & 2 & $2.4 \%$ \\
Results attached to wrong request & 2 & $2.4 \%$ \\
form, i.e., released to wrong client & & \\
Illegible results & 1 & $1.2 \%$ \\
Self request results not easy post & 1 & $1.2 \%$ \\
Dispatched result printout without & 1 & $1.2 \%$ \\
the actual results & & \\
Results released without being & 1 & $1.2 \%$ \\
reviewed & & $1.2 \%$ \\
Results released without request & 1 & \\
form which was found later & & $1.2 \%$ \\
Specimen type not indicated on the & 1 & $100.0 \%$ \\
\hline request form & & \\
Total & &
\end{tabular}


order entry simplifies test ordering and eliminates a second person from transcribing the orders. Automated phlebotomy tray preparation provides a complete set of labeled blood tubes and labels for hand labeling in a single tray for each patient. Preanalytical robotic workstations automate some of the steps and reduce the number of manual steps involving more people. Barcodes can also simplify specimen routing and tracking. ${ }^{7}$

Similar study done. ${ }^{8}$ reported that the information regarding the details of treating physician was missing in $61.2 \%$ the details of diagnosis was not indicated in $19.1 \%$ whereas in $80.9 \%$ where the diagnosis was mentioned, $37.3 \%$ were abbreviated forms. In total of 151 critical results encountered in their study $19.9 \%$ were not communicated to physicians. Specimen drawn in wrong tube $(20,13.5 \%)$, specimen without request forms $(10,6.8 \%)$, unlabeled specimen $(8,5.4 \%)$, and inadequate/insufficient sample after centrifugation $(8,5.4 \%)$. This is comparable to findings. ${ }^{7}$ who established that the main pre-analytical errors encountered are the missing of patient's names. Most studies also reported that the Pre-analytical phase has the predominant frequency of laboratory errors ranging from $53 \%$ to $84.52 \%$. ${ }^{9}$ An article published in 2007, where they found that most pre-analytical errors result from system flaws and insufficient audit of operators involved in that phase. ${ }^{10}$ The promotion of ideal phlebotomy practices and sample transport procedures is a pre-requisite for the efficacy of laboratory functioning. The dependence on accurate laboratory results for diagnostics makes it mandatory for laboratories to ensure accountability and accuracy of results to negate incorrect diagnosis as a consequence of faulty reporting. ${ }^{11}$

The pre-analytical specimen reception area of Clinical Chemistry, tertiary Hospital, has a high throughput and 24 hour operational therefore it can be challenging to document all the errors.

From the study, the higher proportion of errors occurred at the pre-analytical phase, a significant number of the errors were at the analysis phase while slightest although a significant number of the errors were observed at the post-analysis phase. This is consistent with findings by Hammerling, ${ }^{12}$ who observed that the pre-analytical phase of the whole clinical laboratory testing process is where most of the laboratory errors occur. This is also comparable with findings Hawkins, ${ }^{13}$ who established that $31.6 \%$ to $75 \%$ of Clinical laboratory errors occur in the pre-analytical phase. The Swiss cheese model can be used to highlight and improve measures to reduce preanalytical error. A policy for prediction of erroneous events, such as disseminating operative guidelines and reducing the complexity of error-prone activities. ${ }^{2}$
Most of the analytical errors $(93,81.6 \%)$ occurred due to reagent being out of stock $(33,28.9 \%)$, inadequate sample during test runs $(29,25.4 \%)$, haemolysed samples giving poor results $(13,11.4 \%)$, an haemolysed sample is probably less problematic than sample mismatching or a TAT that is too long in a critical situation. duplication of lab number in different specimens $(12,10.5 \%)$, and IQC not done before test runs $(6,5.3 \%) .{ }^{14}$ The remaining $18.4 \%$ (21) of the analytical phase errors are associated to: icterus causing test interference as a factor (5, 4.4\%); analyzer failure during analytical (4, 3.5\%); contradicting results upon re-testing (3, 2.6\%); improper sample storage conditions (3, 2.6\%); wrong specimen analyzed (2,1.8\%); lipemic. Applying the above formula the minimum sample size obtained is 346 . lipemic causing test interference as a factor $(2,1.8 \%)$; name on specimen container and request form not tallying (1, $0.9 \%)$; and test requested not clear $(1,0.9 \%)$.

The study established that $82.1 \%$ (69) of the analytical errors were attributed to: uncollected results $(25,29.8 \%)$; critical values $(17,20.2 \%)$; results lost $(16,19.0 \%)$; and unreviewed results $(11,13.1 \%)$. These findings are consistent with those reported by Plebani. ${ }^{15}$ Which established that the common errors in the post-analytical phase include: wrong validation, results that are not reported, delayed or reported wrong providers, and incorrect results reported because of post-analytical data entry errors as well as transcription errors.

The following errors were not reported at all in the analytical phase: calibration error; lab incident/occurrence/ accidental spillage; EQA/PT not done; and IQC not done. This is consistent with findings by Hammerling, ${ }^{12}$ who highlighted that laboratories have invested heavily on establishing quality results in analytical tests by putting in place internal quality controls (IQC) as well as external quality assessment (EQA). However, for this study, there were 6 incidences where the IQC was not done before test runs.

The total Post-analytical errors captured during the study were $(24.3 \%)$, our study agrees with Plebani. ${ }^{1}$ Study where they found that $23.1 \%$ are post-analytical. Majority of the post-analytical errors in our study were uncollected results 25(29.8\%), therefore uncollected results carried the highest proportion of percentage attributed due to lack of clinic or ward destiny where the results need to be delivered and some laboratory request forms indicated the results to be sent to "self", no clinic or ward in the Hospital with such address. These shows that approximately $30 \%$ of clients do not get back their results and are being managed either empirical or clinically rather than rapidly confirmed diagnoses with targeted curative 
treatment and cost savings. Critical values 17(20.2\%) were not communicated to the clinicians in a timely manner, while studies by Goldschmidt and Lent ${ }^{16}$ reported 23\% delay in treatment due to delay in delivery of quality result to the clinician. Sixteen $(19.0 \%)$ constitute results lost, 11(13.1\%) comprised of un-reviewed results this is attributed by one laboratarian or medical laboratory officer on duty alone. In this study the remaining 18.4\% (21) of the post-analytical phase errors are associated to: wrong test done on specimen $(3,3.6 \%)$; none adherence to turn around time $(2,2.4 \%)$; not all requested tests done $(2,2.4 \%)$; results attached to wrong request form $(2,2.4 \%)$; illegible results $(1,1.2 \%)$; self request results not easy post $(1,1.2 \%)$; dispatched result printout without the actual results $(1,1.2 \%)$; results released without being reviewed $(1,1.2 \%)$; results released without request form which was found later $(1,1.2 \%)$; and specimen type not indicated on the request form $(1,1.2 \%)$. However, the following errors were not reported at all in the postanalytical phase: transcription error; results not posted; and leaking specimen/broken container.

All the pre-analytical, analytical and post analytical errors had an impact to the client or the patient either directly or indirectly, in terms of service delivery, quality health care and cost effect. Most of the patients $40(27.0 \%)$ did not get back their results due request forms lacking address i.e. ward or clinic, and this prolonged the turnaround time for the results due to wasted time in tracing the address, some results took more than a month to reach the clinician or the patients file. And therefore this prolonged the patient stay in the Hospital and quality health care management, and this agrees with Cavenaugh. ${ }^{17}$ whereby he stated that the possible impact of errors on patient care include : no effect, repeated testing, extra clinician appointments, unnecessary medical procedures and therapy, increased duration of hospitalization, disability, and death.

In the three months study a total of $8(5.4 \%)$ unlabeled specimen was received and rejected in the laboratory register. That means another specimen for the eight patients had to be recollected and brought again to the laboratory for analysis.

Majority of the inadequate samples 29 (25.4\%) especially pediatric specimens (ward and clinics) had to be repeated again, same also to the haemolysed 13(11.4\%). Most of the pediatrics are from children aged a day to three years. Adequate blood sample is highly necessary in Clinical laboratory for Clinical analysis and reliable results.

In the pre-analytical phase redrawing or recollection of specimens' increases risks to the patient and in turn increases the expense of care and affects the clinician ability to efficiently treat the patient without undue delay. Clinical laboratory errors also prolong the patient stay in the Hospital.

Most of the Clinical laboratory errors impacts majorly on the patient and the institution budget. Any repeat requires new request forms and new specimen container and a lot of personnel effort to collect the specimen. All errors have consequences and impacts on the cost of the Hospital budget, and hence interfering with the Hospital Mandate and obligations.

\section{CONCLUSION}

The study concludes that pre-analytical, analytical, and post analytical errors are errors that compromise the quality of laboratory service delivery, which impacts on the patient management and diagnosis. Clinical laboratory errors can be minimized if due diligence, professionalism is adhered in the laboratory and requires team work, involving collaboration, coordination, continuous Quality improvement plan for each error identified.

\section{RECOMMENDATION}

There is need for Clinical Laboratory Process Mapping, Process Standardization. Any error occurring in the laboratory at any phase must be documented, disclosed promptly, preventive action and corrective action executed immediately. Also routine and scheduled Clinical laboratory audits in detecting the type of error and in improving Clinical performance. Lastly, Implementing Laboratory Information System (LIS) will minimize and capture Clinical Laboratory errors that compromise quality Laboratory service delivery.

\section{REFERENCES}

1. Plebani M. Laboratory errors: How to improve pre- and postanalytical phases? Biochemia Medica 2007; 17(1):5-9.

2. Malay R, Kyu N and John B. Analysis of Preanalytical Nonconformance in a Medium- Sized Private Pathology Laboratory. Am J Clin Pathol 2014; 142:872-876.

3. International Standards Organization: Medical laboratories -ISO 15189:2012; Requirements for quality and competence. Geneva 2012; (3rd edition).

4. Gershy-Damet G-M, Rotz P, Cross D, Belabbes el H, Cham F, Ndihokubwayo JB, et al. The World Health Organization African Region Laboratory accreditation process: Improving quality of laboratory systems in the African region. Am J Clin Pathol 2010; 134(3):393-400.

5. SLIPTA Checklist: Stepwise Laboratory Quality Improvement Process towards Accreditation 2015; 2:1-49.

6. Yao K, McKinney B, Murphy A, Rotz P, Wafula W, Sendagire H, et al. Improving quality management systems of laboratories in developing countries: An innovative training approach to accelerate laboratory accreditation. Am J Clin Pathol 2010; 134 (3) 401-409. 
7. Da Rin G. Pre-analytical workstations: a tool for reducing laboratory error. Clinical Chim Acta 2009; 404(1):68-74.

8. Nutt L. Annalise EZ and Rajiv TE. Incomplete Laboratory request forms: the extent and impact on critical results at a tertiary Hospital in South Africa. Ann Clin Biochem 2008;45:463-466.

9. Jacobsz LA, Zemlin AE, Roos MJ and Erasmus RT. Chemistry and Haematology sample Rejection and Clinical impact in a Tertiary Laboratory in Cape Town. Clin Chem Lab Med 2011; 49:2047-2050

10. Lippi G, Banfi G, Buttarello M, Ceriotti F, Daves M, Dolci A, et al. Recommendation for detection and Management of unsuitable samples in Clinical Laboratories. Clin Chem Lab Med 2007; 45:728-736.

11. Patra S, Mukherjee B and Das AK. Pre-analytical Errors in the Clinical Laboratory and how to minimize them.2013; www.jijbio.com.
12. Hammerling JA. A Review of Medical Errors in Laboratory Diagnostics and Where We Are Today. Lab Med 2012; 43(2):41-44

13. Hawkins R. Managing the Pre- and Post-analytical Phases of the Total Testing Process. Lab Med 2012; 32(1): 5-16.

14. Bonini P, Plebani M, Ceriotti F and Rubboli F. Errors in laboratory medicine. Clin Chem 2002; 48:691-698.

15. Plebani M. Errors in Clinical laboratories or errors in laboratory medicine? Clinical chemistry laboratory medicine 2006; 44(6): 750-759.

16. Goldschmidt HMJ and Lent RW. Gross errors and work flow analysis in the clinical laboratory. Klin Biochem Metab 1995; 3:131-140.

17. Cavenaugh EL. A method for determining costs with Laboratory error. Am J Public Health 1981; 71:831-834.

Authors Contribution:

KKK - Concept and design of study, data analysis reviewed the literature, critical revision of manuscript; SKW - Concept and design of study, reviewed the literature; JHO - Review of literature, design of study; CSK - Study design and concept, data analysis reviewed the literature, critical revision of the manuscript. Source of Support: Nil, Conflict of Interest: None declared. 